\title{
A von Hamos spectrometer for in situ sulfur speciation by non-resonant sulfur K $\alpha$ emission spectroscopy
}

Received 00th January 20xx, Accepted 00th January 20xx

DOI: $10.1039 / x 0 x \times 00000 x$
Dzulija Kuzmenko a,b, Urs Vogelsang ${ }^{a}$, Stephan Hitz ${ }^{a}$, David Müllera, Joanna Czapla-Masztafiak ${ }^{c}$, Christopher Milne ${ }^{a}$, Jakub Szlachetko ${ }^{c *}$, Maarten Nachtegaal ${ }^{a *}$

A von Hamos geometry based wavelength dispersive spectrometer combined with an in situ reactor cell has been developed to measure non-resonant sulfur Ka emission for the in situ speciation of low concentrations of sulfur. The spectrometer operates at $15 \mathrm{~cm}$ focusing radius, is equipped with a curved $\mathrm{Si}(111)$ crystal and a position sensitive detector, and is capable of achieving an energy resolution of $0.31 \mathrm{eV}$. We present the details of the spectrometer and dedicated sample-cell design to study chemical reactions in situ. The spectrometer capabilities are exemplified by an in situ study of sulfur speciation during $\mathrm{H}_{2} \mathrm{~S}$ poisoning of $\mathrm{SiO}_{2}$ supported Ru nanoparticles performing $\mathrm{CO}$ methanation.

\section{Introduction}

Knowing the sulfur speciation is important in many areas of chemistry and biology. The speciation can be qualitatively and in some cases quantitatively studied by, for example, infra-red $(\mathrm{IR})^{1}$, Raman ${ }^{2}$ and X-ray photoelectron spectroscopy (XPS). ${ }^{3}$ In addition, X-ray absorption (XAS) and X-ray emission (XES) spectroscopies can also be applied to study sulfur speciation in situ. 4,5 XAS and XES provide complimentary information since XAS reflects the density of unoccupied electronic states while XES reflects the transitions from occupied states to the empty core hole $(2 p \rightarrow 1 s$ in the case of $K \alpha)$. K $\alpha_{1,2}$ emission lines primarily reflect the charge density of the sulfur atom, have a simple spectral shape and only the position of the K $\alpha$ emission lines as well as the ratio of $K \alpha_{1}$ to $K \alpha_{2}$ intensity changes for different sulfur oxidation states. ${ }^{6}$ The XAS spectra are not only reflecting the charge density of the sulfur atom, but in addition influenced by orbital hybridization effects which makes the spectral shape complex and feature-rich.7,8 Although these properties can potentially reveal important structural information, when only oxidation state is of interest and/or no exact reference compounds are available, XES provides a reliable and simple way to determine sulfur speciation via a linear combination of $K \alpha$ doublets of various sulfur oxidation state references. Another factor that influences the accurate assignment of the sulfur oxidation state is that XAS, where the incident energy is scanned around the S K-edge, suffer of strong self-absorption effects ${ }^{9}$ that significantly modify the relative ratios of absorption features making in many cases chemical identification challenging or often even impossible. In case of non-resonant XES measurements, self-absorption effects are

\footnotetext{
a. Paul Scherrer Institut, $\mathrm{CH}-5232$ Villigen PSI, Switzerland

b. Department of Chemistry and Applied Biosciences, ETH Zürich, Vladimir-PrelogWeg 1-5, 8093 Zürich, Switzerland

Institute of Nuclear Physics, Polish Academy of Sciences, 31-342 Krakow, Poland *Corresponding authors: maarten.nachtegaal@psi.ch
}

still present, however, when all non-resonant XES spectra are collected at the same incident energy, self-absorption does not influence the measured spectral shape or relative spectral intensities, but only affects the overall yield. ${ }^{10}$ In addition, the incident X-ray energy for non-resonant XES, in contrast to XAS, can be well above the absorption edge. Hence, non-resonant XES does not require access to soft $\mathrm{X}$-rays and can thus be measured at any hard X-ray beamline. Finally, not only the chemical composition can be deduced from spectral analysis of the $S$ XES spectra, but also relative changes in sulfur concentration (for example the absorption or desorption of sulfur species) can be monitored, since the XES signal is directly proportional to the number of sulfur atoms in the beam path.

For measuring non-resonant X-ray emission spectra either wavelength or energy dispersive spectrometers (WDS and EDS) are typically used. To discriminate the energy of the emitted photons, EDS employs solid state detectors which generally have a low energy resolution (on the order of $150 \mathrm{eV}$ or worse). ${ }^{11}$ WDS have a crystal for wavelength dispersion (governed by Bragg's law) which ensures higher energy resolution reaching the natural linewidths of the measured spectral lines (typically 1-4 eV). ${ }^{12}$ To improve the detection efficiency, spectrometers based on focusing bent crystals have been developed. However, bending of the crystal decreases the energy resolution. ${ }^{13}$ The most popular focusing spectrometer geometries are Johann ${ }^{14}$, Johansson ${ }^{15}$ and von Hamos ${ }^{16}$ For the former two geometries, the emission source is usually placed in a circle with the radius half of the crystal bending radius, called Rowland circle. The radiation diffracted by the crystal will converge on the source mirror image point on the Rowland circle, where a photon-counting detector is places. To scan through the energy range, a concerted movement of the crystal and the detector is performed. ${ }^{11}$ Alternatively, by placing the source (sample) inside the Rowland circle and using a position sensitive detector, Johansson spectrometer can also measure the whole emission spectrum in a single acquisition. ${ }^{17}$ The crystal used for Johansson geometry, in contrast to crystals for

This document is the accepted manuscript version of the following article:

Kuzmenko, D., Vogelsang, U., Hitz, S., Mü11er, D., Clark, A. H., Kinsche1, D., Czapla-Masztafiak, J., Milne, C., Szlachetko, J., \& Nachtegaal, M. (2019). A von Hamos spectrometer for: in situ sulfur speciation by non-resonant sulfur $K \alpha$ emission spectroscopy. Journal of Analytical Atomic spectrometry, 34(10), 2105-2111.https://doi.org/10.1039/c9ja00195f 
Johann geometry, is not only bent but its surface is also ground to a Rowland circle radius for precise focusing. In von Hamos geometry, the crystal is only bent in one direction for focusing, while in the other direction the crystal is flat and thus disperses the energy. This has as advantage that the energy resolution is often superior to that of the two other geometries and that the bending radius and with it the diameter of the Rowland circle can be kept small. In the von Hamos geometry the source and detector are placed on the axis of the cylinder of the bent crystal, with the crystal to the source-detector plane distance equal to the crystal bending radius. This geometry provides spatial dispersion of the spectrum allowing to acquire the whole spectrum in one shot. ${ }^{11}$

There are a few X-ray emission spectrometers for S XES reported in the literature. Kavčič et al. ${ }^{18}$ reported a Johansson type spectrometer for the tender (1.6-6.5 keV) X-ray range. The Rowland circle radius was $50 \mathrm{~cm}$ and the sample was placed inside the Rowland circle $27 \mathrm{~cm}$ from the Si (111) crystal. A charge-coupled device (CCD) was used as a position sensitive detector. Alonso Mori et al. have reported $\mathrm{S} \mathrm{K} \alpha^{7}$ and $\mathrm{K} \beta^{19}$ of various reference compounds using this spectrometer geometry. The energy resolution achieved was $0.44 \mathrm{eV}$. The same spectrometer design was also used to measure $\mathrm{S} K \alpha$

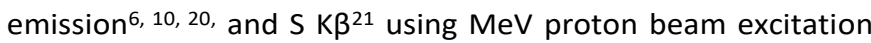
from a proton tandem accelerator, achieving a final experimental resolution of $0.45 \mathrm{eV}$. An operando study of $\mathrm{Li}-\mathrm{S}^{4}$ and $\mathrm{Mg}-\mathrm{S}^{22}$ batteries using sulfur resonant inelastic X-ray scattering (RIXS) was also carried out using the above described Johansson type spectrometer, where the incident energy is scanned around the absorption edge and at each incident energy the corresponding sulfur Ka emission line measured. For RIXS, in contrast to non-resonant XES, the core electron is resonantly excited to the absorption threshold not to the highenergy continuum, making the spectrum dependent on the incident photon energy. ${ }^{23}$ Kavčič et al. ${ }^{24}$ have measured sulfur $\mathrm{K} \beta$ emission using a $\mathrm{Cr} \mathrm{X}$-ray tube as an X-ray source. The spectrometer was designed in the von Hamos geometry with a $\mathrm{SiO}_{2}$ (110) cylindrically bent crystal to a radius of $254 \mathrm{~mm}$. The energy resolution achieved was comparable to the natural linewidth of the $\mathrm{S} \mathrm{K \alpha}$ emission line $(0.64 \mathrm{eV})$. Holden et al.8,25 have designed a Johann geometry spectrometer with a $10 \mathrm{~cm}$ diameter Rowland circle and complementary metal-oxidesemiconductor (CMOS) X-ray camera, achieving a resolution of $0.7 \mathrm{eV}$. The spectrometer utilizes the dispersive refocusing Rowland (DRR) circle geometry that allows the use of an unfocused X-ray beam. This instrument can be used with a lowpowered X-ray tube, as well as at synchrotrons or X-ray free electron lasers.

Here we present the design and application of a von Hamos geometry based spectrometer combined with an in situ cell designed specifically for studying heterogeneous catalysts in the presence of reactive gases at atmospheric pressures and high temperature, to determine the in situ speciation of sulfur. In the shown example, we were able to detect sulfur adsorption from a $100 \mathrm{ppm} \mathrm{H}_{2} \mathrm{~S}$ gas stream by the surface of $\mathrm{SiO}_{2}$ supported $\mathrm{Ru}$ nanoparticles and assign the oxidation state of adsorbed sulfur as a mixture of $\mathrm{S}^{-1}$ and $\mathrm{S}^{-2}$ species.

\section{Spectrometer and cell design}

A scheme of the von Hamos geometry ${ }^{13}$ projected along the dispersive plane is shown in Fig. 1. Briefly, a cylindrically bent crystal diffracts X-ray photons emitted by the sample after irradiation to a position sensitive detector. The position of the

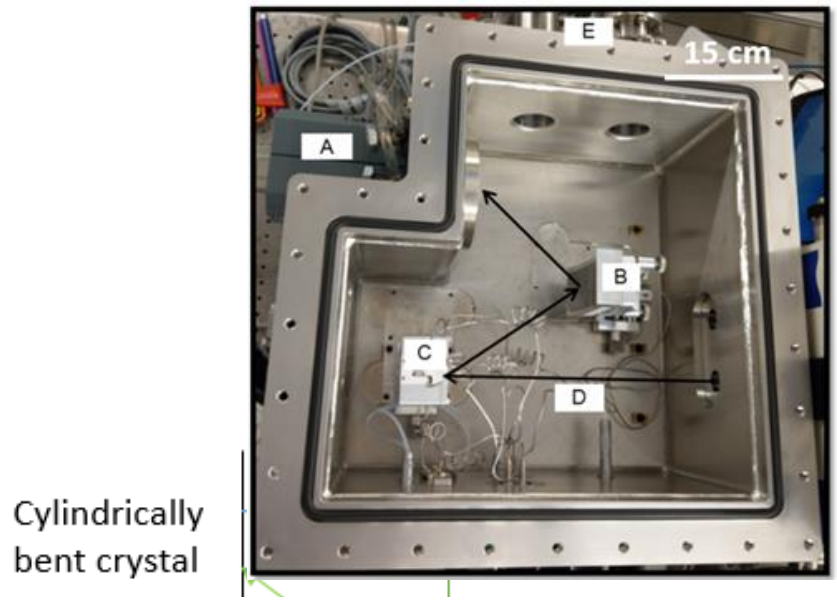

Fig. 2: X-ray emission spectrometer - A: CCD detector, B: Si (111) $15 \mathrm{~cm}$ bendin radius crystal, C: In situ cell, D: X-ray path, E: Turbo pump.

\section{Sample}

Fig. 1: von Hamos geometry projected along the dispersive plane (top view) for sulfur Ka emission measurement

diffracted X-rays on the detector correlates to the energy, according to Bragg's law:

$$
2 d \sin (\vartheta)=\mathrm{n} \lambda
$$

where $2 \mathrm{~d}$ is the crystal interplanar distance, $\vartheta$ is the scattering angle, $\lambda$ is the emission radiation wavelength and $n$ is an integer, indicating the diffraction order. For sulfur K $\alpha$ emission, which has an energy of $2.307 \mathrm{keV}$, we have chosen a Si (111) crystal with an interplanar spacing $d=6.27 \AA .{ }^{13}$ Taking $n=1$, the scattering angle $\vartheta$ is $58.98^{\circ}$ for this crystal. The distances from the sample to the center of the crystal and from the detector to the crystal center are equal to the crystal curvature radius $(\mathrm{R}=15$ $\mathrm{cm})$ multiplied by $\cot (\vartheta), 9.02 \mathrm{~cm}$.

In addition, the center of the Si (111) crystal within the current geometry (Fig. 1) will also diffract triple the energy of the sulfur $\mathrm{K} \alpha$ emission (when $\mathrm{n}=3$ in Bragg's law), i.e. $6.921 \mathrm{keV}$. For this reason, we have chosen the incident energy for non-resonant XES to be around $6.9 \mathrm{keV}$ since this allows us to observe both sulfur K $\alpha$ emission and elastic scattering of the incident beam in the same spectrum. The elastic scattering is used to calibrate the energy axis and to crosscheck experimental stability and reproducibility; a pixel-to-energy calibration curve is constructed by measuring each sample at different excitation energies in the range from 6.8 to $7 \mathrm{keV}$. Also the width of the elastic scattering (at incident energy of $6.869 \mathrm{keV}$ ) peak was used to determine the energy resolution of the spectrometer in the first diffraction order.

The whole spectrometer is placed in a stainless steel chamber (Fig. 2) which is evacuated by a turbo pump to $10^{-3}-10^{-4} \mathrm{mbar}$. We have 
chosen a $15 \mathrm{~cm}$ radius crystal as the best compromise between efficiency of the setup (solid angle) and the spectrometer resolution. For crystal manufacturing, a $100 \mu \mathrm{m}$ thick Si (111) wafer (50 $\mathrm{mm}$ in dispersion and $100 \mathrm{~mm}$ in focusing) was glued on a curved aluminum substrate. The crystal was placed on a motorized stage allowing for fine-tuning of the crystal position in the focusing direction. The $\mathrm{X}$-ray radiation diffracted by the crystal is detected by a charge-coupled device (CCD) detector (Andor DO440, 2048 x 512 array with $13.5 \times 13.5 \mu \mathrm{m}$ pixels), which was thermoelectrically cooled to $-30^{\circ} \mathrm{C}$. The chip length allows measuring the energy range from 2.255 to $2.348 \mathrm{keV}$ in a single acquisition. An aluminized $6 \mu \mathrm{m}$ thick Mylar window was placed in front of the CCD chip to prevent visible light from reaching the chip.

The here developed stainless steel in situ cell (Fig. 3) is inspired by the design of a previously published ${ }^{26}$ in situ cell. The cell is equipped with a heating element able to reach $600^{\circ} \mathrm{C}, 4$ thermocouples and is connected to $1 / 16$ inch tubes for gas flow. A $7 \mu \mathrm{m}$ thin Kapton film was used as window material. In addition, a special casing (heat protective cover) was developed to protect the surroundings of the cell from overheating. The cell also has three ceramic bearings designed to minimize its vertical and horizontal expansion during heating and thus keep the sample in place during heating and cooling. Finally, the cell does not have an aperture at the rear since only fluorescence is measured. The cell allows for in situ experiments, feeding

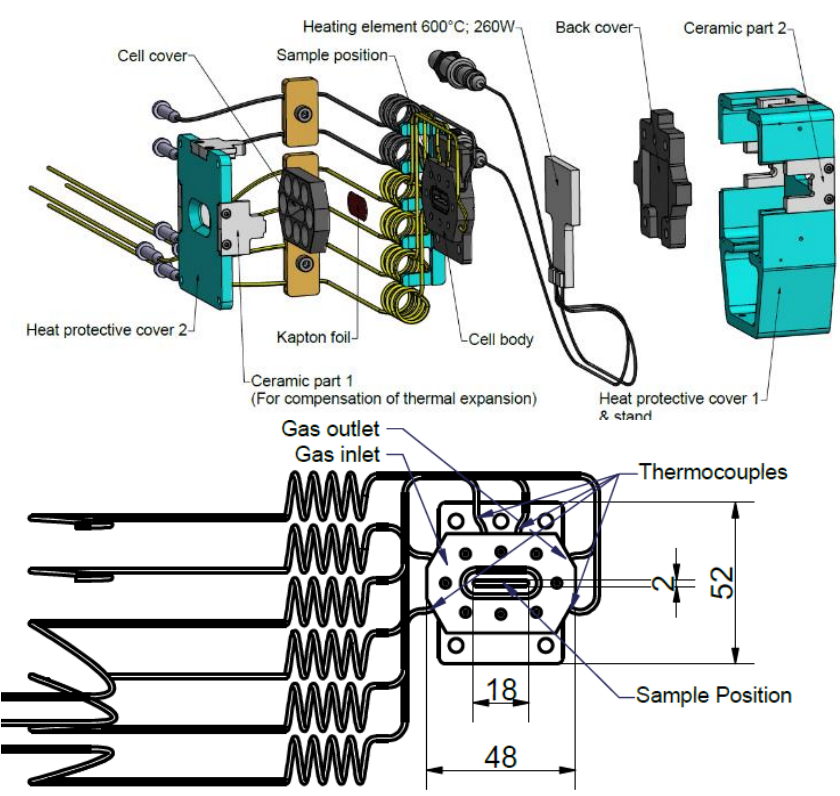

Fig. 3: In situ cell design: all components (top) and cell body with dimensions in $\mathrm{mm}$ (bottom)

reactive gases at atmospheric pressure, even though the cell itself is placed in vacuum.

\section{Experimental}

The spectrometer was installed at the SuperXAS beamline of the Swiss Light Source (SLS), Villigen, Switzerland. The SLS operated at $400 \mathrm{~mA}$ and $2.4 \mathrm{GeV}$. The polychromatic beam from the 2.9
Tesla superbend collimated by a collimating Si-coated mirror at $2.5 \mathrm{mrad}$ and subsequently monochromatized by a Si (111) channel-cut crystal. Downstream of the monochromator, a Ptcoated torroidal mirror was used to focus the incident $x$-rays to a spot size of $100 \times 100 \mu \mathrm{m}^{2}$ at the sample position. The incoming $\mathrm{X}$-ray energy was calibrated by using a Fe foil at the start of the experiment.

Reference compounds $\left(\mathrm{Ag}_{2} \mathrm{~S}, \mathrm{FeS}_{2}, \mathrm{~S}, \mathrm{Na}_{2} \mathrm{SO}_{3}\right.$ and $\left.\mathrm{Na}_{2} \mathrm{SO}_{4}\right)$ were purchased from Sigma Aldrich and used as received. The powder was crushed using a pestle and mortar and subsequently pressed in a $0.5 \mathrm{~cm}$ diameter pellet without addition of any binder. The emission spectrum was recorded for 60 seconds, apart from the elemental $\mathrm{S}$ sample, where the acquisition time was 5 seconds.

For the in situ poisoning experiment, $10 \mathrm{mg}$ of $3 \% \mathrm{Ru} / \mathrm{SiO}_{2}$, prepared according to reference ${ }^{27}$, were placed in the cell and after the evacuation of the chamber the cell was heated to $360{ }^{\circ} \mathrm{C}\left(10^{\circ} \mathrm{C} / \mathrm{min} \mathrm{ramp}\right)$ while He was flowing through the cell. Subsequently, the catalyst was treated with a $2.5 \% \mathrm{H}_{2}, 0.5 \% \mathrm{CO}$ and $100 \mathrm{ppm} \mathrm{H}_{2} \mathrm{~S}$ mixture for 30 minutes at $360^{\circ} \mathrm{C}$ after which the gas flow was switched to $\mathrm{He}$ and the emission signal was recorded for 1320 seconds. The cell is operated at an ambient

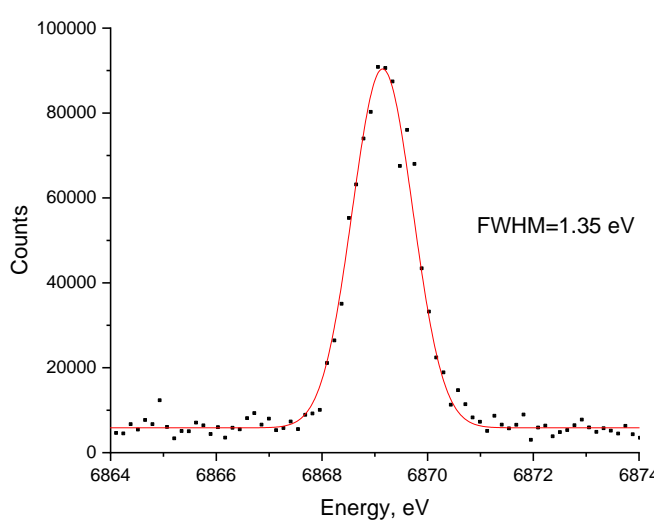

pressure, which allows connecting a mass spectrometer for online monitoring of the gas composition at the cell outlet.

The acquired raw XES spectra were normalized by the total area under the peak using an in house written software. The normalized spectra were then fitted using a pseudo-Voigt peak function, which has Lorentzian and Gaussian function contributions. The Lorentzian function is used to account for the core-hole lifetime broadening and the Gaussian function to account for the instrumental broadening.

\section{Energy resolution and sulfur speciation}

The energy resolution is a convolution of the spectrometer and beamline monochromator resolution when measured by means of elastic scattering peak. The spectrometer resolution depends on the detector pixel size, crystal curvature radius and the incoming $\mathrm{X}$-ray beam spot size. With our configuration, a total energy resolution of ca. $1.35 \mathrm{eV}$ at $6869 \mathrm{eV}$ (with $\mathrm{Si}(111)$ monochromator and $\mathrm{Si}(333)$ diffraction order in the spectrometer) was achieved, as determined from the FWHM of Fig. 4: The elastic scattering peak fitted with Gaussian peak function giving a FWHM of $1.35 \mathrm{eV}$ 
the Gaussian peak function fitting of the elastic peak (Fig. 4). Since the relative energy resolution of the $\mathrm{Si}(111)$ monochromator is equal to $1.4 * 10^{-4}$, the contribution of the incident X-ray bandwidth to the total energy resolution is $1.4 * 10^{-4} * 6869=0.96 \mathrm{eV}$. The total experimental resolution is a root sum of squares (assuming Gaussian functions for incident beam and spectrometer contributions):

Total resol. $=\sqrt{\text { Incident beam resol. }{ }^{2}+\text { Spectrometer resol. }^{2}}$

Thus the spectrometer resolution at $6869 \mathrm{eV}$ is $0.94 \mathrm{eV}$. To calculate the spectrometer resolution at the first diffraction order, we need to divide the resolution of the third diffraction order by 3 , which is $0.94 / 3=0.31 \mathrm{eV}$. This is well below the $\mathrm{S} \mathrm{K} \alpha$ lifetime broadening of $0.59 \mathrm{eV}^{7}$, and thus allows to distinguish different sulfur oxidation states.

To check if it is possible to distinguish various sulfur oxidation states with the present spectrometer configuration, we have measured 5 references compounds: $\mathrm{Ag}_{2} \mathrm{~S}, \mathrm{FeS}_{2}, \mathrm{~S}, \mathrm{Na}_{2} \mathrm{SO}_{3}$ and $\mathrm{Na}_{2} \mathrm{SO}_{4}$ with sulfur formal oxidation states of $-2,-1,0,+4$ and +6 respectively (Fig. 5). As shown, the $K \alpha_{1,2}$ peaks are clearly distinguishable and the positions are in a good agreement with literature data (Table 1)

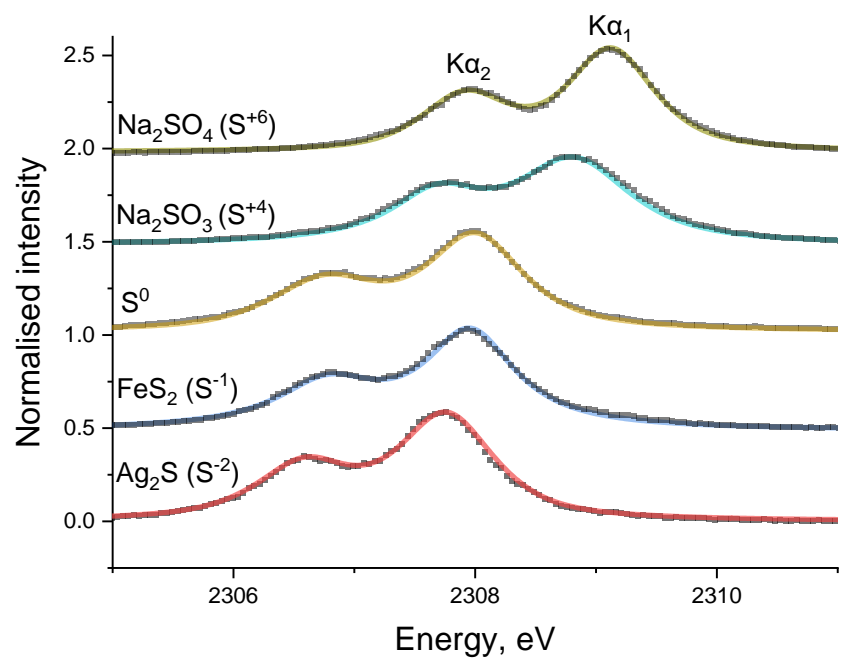

Fig. 5: Sulfur $K \alpha_{1,2}$ emission spectra and the corresponding pseudo-Voigt peak function fit of reference compounds: $\mathrm{Ag}_{2} \mathrm{~S}, \mathrm{FeS}_{2}, \mathrm{~S}, \mathrm{Na}_{2} \mathrm{SO}_{3}$, and $\mathrm{Na}_{2} \mathrm{SO}_{4}$. The spectra are normalized by the total peak area and offset by 0.5 in y-axis for clarity.

Table 1: Reference compounds $\mathrm{K} \alpha_{1}$ emission line position

\begin{tabular}{|c|c|c|}
\hline Compound & $\mathrm{K \alpha}_{1}, \mathrm{eV}$ (this work) & $\mathrm{K \alpha}_{1}, \mathrm{eV}$ (literature) \\
\hline $\mathrm{Ag}_{2} \mathrm{~S}$ & 2307.77 & $2307.7-2307.8\left(\mathrm{~S}^{-2}\right.$ range) \\
\hline $\mathrm{FeS}_{2}$ & 2307.96 & 2307.86 \\
\hline $\mathrm{S}$ & 2308.0 & 2307.89 \\
\hline $\mathrm{Na}_{2} \mathrm{SO}_{3}$ & 2308.82 & 2308.88 \\
\hline $\mathrm{Na}_{2} \mathrm{SO}_{4}$ & 2309.12 & 2309.12 \\
\hline
\end{tabular}

\section{Application to in situ studies}

Fig. 6 shows the $\mathrm{Ka}_{1,2}$ emission spectrum of $\mathrm{H}_{2} \mathrm{~S}$ poisoned $\mathrm{Ru} / \mathrm{SiO}_{2}$ (treated with a $2.5 \% \mathrm{H}_{2}, 0.5 \% \mathrm{CO}$ and $100 \mathrm{ppm} \mathrm{H}_{2} \mathrm{~S}$ mixture for 30 minutes at $360^{\circ} \mathrm{C}$ ), recorded under $\mathrm{He}$ with a total integration time of 1320 seconds. The spectrum was fitted with a linear combination of $80 \% \mathrm{~S}^{-2}$ and $20 \% \mathrm{~S}^{-1}$ references pseudo-Voigt peak function fits. Hence we can assign the oxidation state of sulfur in the poisoned sample as a mix of -1 and -2 , which is in line with what is expected for the sulfur adsorbed on the surface of the metal under a reductive treatment. ${ }^{28}$ This experiment also exemplifies that low sulfur concentrations can be measured with the current set up. Assuming there is no gradient of sulfur concentration along the catalyst bed and that we have reached the saturation coverage of $\mathrm{S}$ on the surface of the Ru nanoparticles, there is on average about one sulfur atom per two surface metal atoms. ${ }^{29}$ With a Ru loading of $3 w t \%$ and dispersion of $0.4,1.2 w t \%$ of the Ru atoms are surface atoms. Taking $n$ (Ru surface) $=1 / 2 n(S$ adsorbed), the maximum concentration of sulfur is expected to be around 0.19 wt\%.

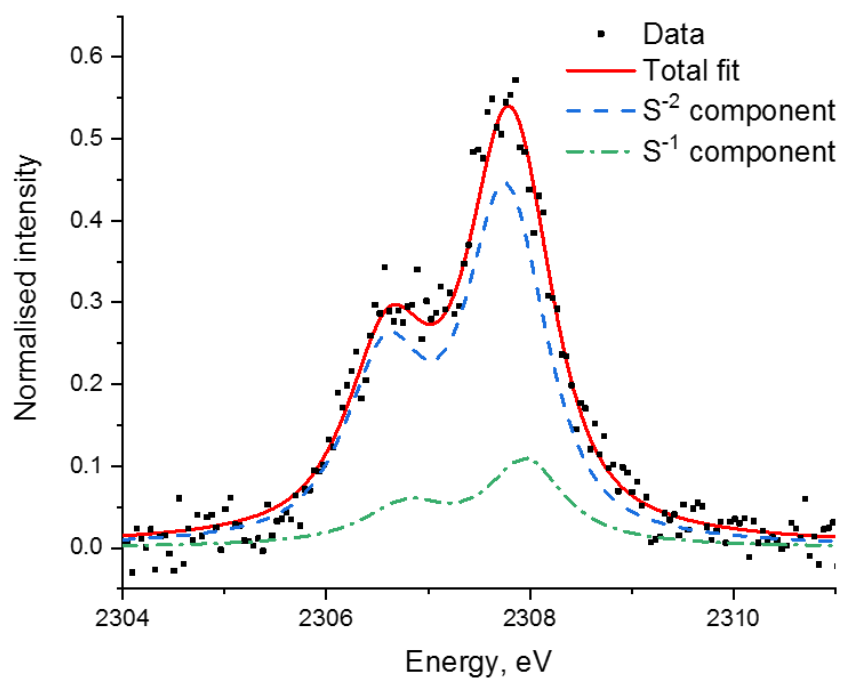

Fig. 6: Sulfur $\mathrm{K}_{1,2}$ emission and pseudo-Voigt peak function fit $\left(80 \% \mathrm{~S}^{-2}\right.$ and $\left.20 \% \mathrm{~S}^{-1}\right)$ of $\mathrm{Ru} / \mathrm{SiO}_{2}$ treated with $2.5 \% \mathrm{H}_{2}, 0.5 \% \mathrm{CO}$ and $100 \mathrm{ppm} \mathrm{H}_{2} \mathrm{~S}$ mixture for 30 minutes at $360^{\circ} \mathrm{C}$. Integration time $1320 \mathrm{sec}$.

\section{Conclusion and outlook}

Here we presented the development of a von Hamos based Xray emission spectrometer together with an in situ cell that allows for non-resonant XES studies to determine the speciation of sulfur under in situ conditions of operating a catalyst.

In the future, it is envisioned to combine this spectrometer with an X-ray tube as a laboratory X-ray source. Provided that a relatively small $X$-ray spot size $(0.1 \mathrm{~mm})$ can be achieved on the sample position, the same resolution and similar count rates can potentially be achieved ${ }^{25}$ that could make the experiments more accessible and easier to carry out for a larger community and for experiments where no time resolution is needed. In 
addition, by making slight adjustments to the set-up we can measure X-ray emission of other light elements, such as phosphorous Ka. Finally, since we observed a decent count rate in a reasonable timeframe for both pure target reference compounds and the in situ sample, the measurement of the ca. 20 times weaker ${ }^{30}$ sulfur $K \beta$ emission line is also plausible with this spectrometer.

\section{Conflicts of interest}

There are no conflicts to declare

\section{Acknowledgements}

This research project is funded by Swiss National Science Foundation and supported by the Energy System Integration platform (ESI) and by the Swiss Innovation Agency Innosuisse as part of the Swiss Competence Center for Energy Research SCCER BIOSWEET. The Swiss Light Source is acknowledged for the provision of beamtime at the superXAS beamline. We also acknowledge the National Science Centre, Poland (NCN), for partial support under grant no. 2016/21/D/ST4/00378.

\section{Notes and references}

1 C. C. Chang, J. Catal., 1978, 53, 374-385.

2 T. Wilke, X. Gao, C. G. Takoudis and M. J. Weaver, J. Catal., 1991, 130, 62-75.

3 R. P. W. J. Struis, T. J. Schildhauer, I. Czekaj, M. Janousch, S. M. A. Biollaz and C. Ludwig, Appl. Catal. A Gen., 2009, 362, 121128.

4 M. Kavčič, K. Bučar, M. Petric, M. Žitnik, I. Arčon, R. Dominko and A. Vizintin, J. Phys. Chem. C, 2016, 120, 24568-24576.

5 C. F. J. König, P. Schuh, T. Huthwelker, G. Smolentsev, T. J. Schildhauer and M. Nachtegaal, Catal. Today, 2014, 229, 5663.

6 M. Kavčič, A. G. Karydas and C. Zarkadas, Nucl. Instruments Methods Phys. Res. Sect. B Beam Interact. with Mater. Atoms, 2004, 222, 601-608.

7 R. Alonso Mori, E. Paris, G. Giuli, S. G. Eeckhout, M. Kavčič, M. Žitnik, K. Bučar, L. G. M. Pettersson and P. Glatzel, Anal. Chem., 2009, 81, 6516-6525.

8 W. M. Holden, G. T. Seidler and S. Cheah, J. Phys. Chem. A 2018, 122, 5153-5161.

9 F. Jalilehvand, Chem. Soc. Rev., 2006, 35, 1256-1268.

10 M. Petric and M. Kavčič, J. Anal. At. Spectrom., 2016, 31, 450457.

11 P. Willmott, An Introduction to Synchrotron Radiation: Techniques and Applications, Wiley, 2019.

12 M. Kavčič, in X-Ray Spectroscopy, ed. S. K. Sharma, IntechOpen, Rijeka, 2012.

13 J. Szlachetko, M. Nachtegaal, E. de Boni, M. Willimann, O. Safonova, J. Sa, G. Smolentsev, M. Szlachetko, J. A. van Bokhoven, J.-C. Dousse, J. Hoszowska, Y. Kayser, P. Jagodzinski, A. Bergamaschi, B. Schmitt, C. David and A. Lücke, Rev. Sci. Instrum., 2012, 83, 103105.

14 H. H. Johann, Zeitschrift für Phys., 1931, 69, 185-206.

15 T. Johansson, Zeitschrift für Phys., 1933, 82, 507-528.

16 L. v. Hámos, Naturwissenschaften, 1932, 20, 705-706.
17 R. Alonso-Mori, J. Kern, D. Sokaras, T.-C. Weng, D. Nordlund, R. Tran, P. Montanez, J. Delor, V. K. Yachandra, J. Yano and U. Bergmann, Rev. Sci. Instrum., 2012, 83, 73114.

18 M. Kavčič, M. Budnar, A. Mühleisen, F. Gasser, M. Žitnik, K. Bučar and R. Bohinc, Rev. Sci. Instrum., 2012, 83, 33113.

19 R. A. Mori, E. Paris, G. Giuli, S. G. Eeckhout, M. Kavčič, M. Žitnik, K. Bučar, L. G. M. Pettersson and P. Glatzel, Inorg. Chem., 2010, 49, 6468-6473.

20 M. Kavčič, A. G. Karydas and C. Zarkadas, X-Ray Spectrom., 2005, 34, 310-314.

21 M. Kavčič, M. Petric and K. Vogel-Mikuš, Nucl. Instruments Methods Phys. Res. Sect. B Beam Interact. with Mater. Atoms, 2018, 417, 65-69.

22 A. Robba, A. Vizintin, J. Bitenc, G. Mali, I. Arčon, M. Kavčič, M. Žitnik, K. Bučar, G. Aquilanti, C. Martineau-Corcos, A. RandonVitanova and R. Dominko, Chem. Mater., 2017, 29, 95559564.

23 A. Kotani and S. Shin, Rev. Mod. Phys., 2001, 73, 203-246.

24 M. Kavčič, J.-C. Dousse, J. Szlachetko and W. Cao, Nucl. Instruments Methods Phys. Res. Sect. B Beam Interact. with Mater. Atoms, 2007, 260, 642-646.

25 W. M. Holden, O. R. Hoidn, A. S. Ditter, G. T. Seidler, J. Kas, J. L. Stein, B. M. Cossairt, S. A. Kozimor, J. Guo, Y. Ye, M. A. Marcus and S. Fakra, Rev. Sci. Instrum., 2017, 88, 73904.

26 S. Hannemann, M. Casapu, J.-D. Grunwaldt, P. Haider, P. Trüssel, A. Baiker and E. Welter, J. Synchrotron Radiat., 2007, 14, 345-354.

27 K. Furman, D. Baudouin, T. Margossian, K. D. Sabnis, Y. Cui, F. H. Ribeiro and C. Copéret, J. Catal., 2015, 324, 9-13.

28 P. K. Agrawal, J. R. Katzer and W. H. Manogue, J. Catal., 1982, 74, 332-342.

29 C. H. Bartholomew, P. K. Agrawal and J. R. Katzer, eds. D. D. Eley, H. Pines and P. B. Weisz, Academic Press, 1982, vol. 31, pp. $135-242$.

30 http://xdb.Ibl.gov/Section1/Table_1-3.pdf 